\title{
Grundlagen für das Thema Frieden in Baudissins Werk
}

\author{
Claus von Rosen*
}

\begin{abstract}
The spiritual turning point in Baudissin's life is shown in his works »East or West - thoughts about a vital GermanEuropean question «, written during his imprisonment in 1946. He received essential impetus from Wilhelm Röpke's »Civitas Humana « and Emil Brunner's »Justice«. Baudissin's worries were about a general new ethic orientation. Thus, peace on earth has become a decisive normative and objective category of thinking. He views this peace of the individual as »inner peace" as well as a political one, both being interlinked. In the face of a »common peacelessness « he looks for political possibilities of improvement and recommends to focus on the immediate objective, the political concrete. These are the foundations of his further work as the creator of »Inner Leadership« with the model of a »Soldier for Peace« as well as the peace researcher counselling politicians on the strategies of deterrent and cooperative steering of armament.
\end{abstract}

Keywords: Innere Führung, Ernstfall Frieden, Soldat für den Frieden, Kooperative Rüstungssteuerung

$\mathrm{W}$ olf Graf von Baudissin, Schöpfer der Führungsphilosophie der Bundeswehr Innere Führung und Friedensforscher, verstand sich als Vierzigjähriger, d.h. noch nach Ende des Zweiten Weltkrieges, als Jung-Konservativer. Geboren 1907, war er in seinem Elternhaus entsprechend geprägt worden. ${ }^{1} 1926$ trat er als Offizieranwärter in das Infanterie-Regiment 9 der Reichswehr in Potsdam ein, unterbrach diese Ausbildung für drei Jahre zum Studium der Landwirtschaft und tat dann wieder Dienst als Zugführer, Bataillonsadjutant und Regimentsadjutant im IR 9, bis er 1938 zur Generalstabsausbildung kommandiert wurde. Im Krieg in Frankreich und in Afrika als Generalstabsoffizier eingesetzt, kam er 1941 in britische Gefangenschaft und wurde erst in Palästina und danach bis kurz vor seiner Entlassung im Sommer 1947 in Australien gefangen gehalten.

In der Gefangenschaft befasste Baudissin sich intensiv mit der von ihm so genannten "Schicksalsfrage« seiner Zeit. Dazu nahm er Anregungen aus Seminaren und Vorlesungen auf, die er selber im Gefangenenlager gestaltet und gehalten bzw. an denen er als Hörer teilgenommen hatte. Weiter ließ er sich durch diverse Lektüre anregen, die er auf Umwegen über Großbritannien sowie Argentinien angefordert oder sich im Lande selber beschafft sowie - vermutlich nach Ende des Krieges - von Hilfsorganisationen aus der Schweiz erhalten hatte. Insbesondere der tiefe geistige Gedankenaustausch per Brief mit seiner späteren Braut Dagmar Gräfin zu Dohna führte ihn dabei zu wesentlichen Einsichten. ${ }^{2}$ Seine Gedanken fasste er im Laufe des Jahres 1946 zu einer Denkschrift: »Ost oder West - Gedanken zur deutsch-europäischen Schicksalsfrage« zusammen, die er im Winter 1946 in Maschinen-Reinschrift

* Dr. Claus Frhr. von Rosen ist Leiter des Baudissin-Dokumentation-Zentrums bei der Führungsakademie der Bundeswehr.

1 Baudissin, Wolf Graf von: Als aus Neustadt Wejherowo wurde. In: Rudolf Pörtner (Hrsg.): Mein Elternhaus. Ein deutsches Familienalbum. Düsseldorf, Wien 1984, S. 69-78. Baudissin hat in seiner Abschiedsvorlesung von 1986 selber einen kurzen Lebensrückblick gegeben, s. Baudissin, Wolf Graf von und Dagmar Gräfin zu Dohna: ... als wären wir nie getrennt gewesen. Briefe 1941-1947. Bonn 2001, S. $258 \mathrm{ff}$.

2 Wolf Graf von Baudissin: 23-Zeilen-Briefe. Hamburg (Selbstverlag 1994. S.a ders. a.a.O. 2001. In dem Brief vom 10.2.1946 fällt auch der Begriff des JungKonservativen. verfasste. ${ }^{3}$ Sein Denken in dieser Denkschrift »Ost oder West « ist trotz vieler unkonventioneller Gedanken und tieferen Durchleuchtungen der geschichtlichen Gegebenheiten bis in ethische Dimensionen noch deutlich konservativ. ${ }^{4}$

Die Zeit der Gefangenschaft und besonders die Arbeit an der Denkschrift werden dann aber augenfällig zum Wendepunkt in Baudissins Denken, Verstehen und Erkenntnissen, bis dass er sich nun selber als »aufgeklärten Anhänger von Karl Marx« verstand. ${ }^{5}$ Damit stellt sich allgemein die Frage, welche Anregungen aus dieser Zeit für seine späteren Arbeiten zur Inneren Führung und Friedensforschung bedeutsam geworden sind, und hier speziell: wie das Thema Frieden für Baudissins weiteres Denken und Handeln die hervorragende und bestimmende Kategorie geworden ist.

\section{Baudissin-Anknüpfungspunkte bei Emil Brunner}

Während der Arbeit an der Reinschrift von »Ost oder West « stieß Baudissin auf das Buch von Wilhelm Röpke »Civitas Hu-

3 Vrgl. Baudissin 2001 a.a.O. S. 131. Das Original der Denkschrift befindet sich im Baudissin-Nachlass im Baudissin-Dokumentation-Zentrum bei der Führungsakademie der Bundeswehr. Über die Denkschrift wurde erstmalig berichtet in: Claus von Rosen: Ost oder West - „Gedanken zur deutsch-europäischen Schicksalsfrage «. In: Hilmar Linnenkamp, Dieter S. Lutz (Hrsg.): Innere Führung. Zum Gedenken an Wolf Graf von Baudissin. Baden-Baden 1994. - Künftig zitiert: Baudissin 1947, unter Angabe von Kapitel bzw. Abschnitt.

4 Hierzu s. Martin Kutz: Aus den Katastrophen der Geschichte lernen: Über den historischen Ort der Konzeption Baudissins. In: Ders. (Hrsg.): Gesellschaft, Militär, Krieg und Frieden im Denken von Wolf Graf von Baudissin. Band 23 der Reihe: Forum Innere Führung, Baden-Baden 2004, S. 13-24. - Dieses (Jung-)Konservative kann auch manche Bemerkungen in der Denkschrift über den Nationalsozialismus erklären, die bei Baudissins sonst gegenüber dem Nazi-Regime ablehnender Haltung (s.a. ders. 1947, Abschn. 7.2) höchst auffällig sind. So schreibt er in Abschn. 6.4: "Mit dem Ende des Nationalsozialismus ist Deutschland für immer verloren« u.a.: »Das Dritte Reich war ein großartiger Versuch, unsere deutschen und später die europäischen Probleme mit den deutschen Mitteln seiner Zeit zu lösen, zu dem sich jeder wirklich Deutsche bekennen wird; es hatte damit alles Großartige eines solchen kühnen Unterfangens, aber naturgemäß auch die Mängel eines Versuchs, welcher unter schwierigsten Verhältnissen und in einer Zeit unternommen wurde, wie die heute geltenden Regeln bereits morgen überholt sein können.«

5 Baudissin 1947, Abschn. 3.22. S.a. Rosen 1994 a.a.O. S. 113 f. 
mana «. ${ }^{6}$ Röpke seinerseits drückt im Vorwort von Dezember 1943 sein Bedauern aus, dass er erst zum Zeitpunkt des Korrekturlesens auf das Buch »Gerechtigkeit « von Emil Brunner ${ }^{7}$ gestoßen sei, »so dass ich mich einstweilen damit begnügen muss, meine hohe Wertschätzung dieses Werkes und meine wesentliche Übereinstimmung mit dem Verfasser zum Ausdruck zu bringen «. Neben einigen anderen Ergänzungen arbeitet Baudissin im Winter 1946/47 die Gedanken aus beiden Büchern nachträglich als zum Teil umfangreiche Anmerkungen sowie wörtliche Zitate in die Denkschrift ein. ${ }^{8}$ D.h. die Lektüre dieser beiden Autoren hat besonders große Bedeutung für Baudissins Nach-Denken gehabt. Man kann sie daher bildlich als den Initialfunken bezeichnen, der Baudissins jahrelange Vorarbeiten plötzlich zum Lodern gebracht hat.

Die Frage, wieso Brunners Buch »Gerechtigkeit« - und nur dies soll im Weiteren betrachtet werden - diese enorme Wirkung bei Baudissin gezeigt hat, wird von Kutz mit Brunners konservativ-bürgerlichem Gesellschaftsbild erklärt, das dem Baudissins in jener Zeit noch sehr nahe stand. ${ }^{9}$ Dieser Erklärungsansatz verlangte ein gedanklich-emotionales cross-over d.h.: im Konservativen Brunner Anregungen für die eigene Progression zu erfahren und diese dann zu entwickeln, um die eigenen Konservativismen zu überwinden. Dieser nicht undenkbare Grundgedankengang ist aber nicht nur schwerfällig, sondern wäre für Baudissins Gedankenarbeit im abgeschiedenen »Gewächshausdasein « der Gefangenschaft besonders schwierig. ${ }^{10}$

\section{Baudissin ist von Brunner sehr viel direkter angesprochen} worden:

Zum einen hat Brunner als Vertreter eines anthropologischen Ansatzes der dialektischen Theologie, geistig der Bekennenden Kirche und als Lehrer von Gerstenmeier dem Kreisauer Kreis nahestehend, Baudissins tiefe christlich-ethische Gebundenheit und Gedanken unmittelbar angesprochen, wie z.B. aus

6 Wilhelm Röpke: Civitas Humana. Grundfragen der Gesellschafts- und Wirtschaftsreform. Erlenbach-Zürich 1944. Das Exemplar aus Baudissins Nachlass ist gestempelt: »Fonds Européen de Secours aux Ètudiants; 13, rue Calvin; Genève (Suisse) « und handschriftlich gewidmet: »Tartura 1946 F.v.G. <oder: $\mathrm{H}>$ «. Ein Preisvermerk auf dem Umschlag ist mit dem Datum 16.2.45 versehen. Baudissins Arbeitsexemplar aus der Gefangenschaft befindet sich in seinem Nachlass.- In einer Anmerkung zum Abschnitt 2.2. »Begriffe der Politik « in der Denkschrift stellt Baudissin diese »Verspätung « mit Bedauern fest. Dort befasst Baudissin sich mit Röpkes Gedanken zum »idealen modernen Staatsaufbau«; im Abschnitt $3.22 »$ Der soziale und staatliche Aufbau als asiatische Lösung « nimmt er Röpkes Ausführungen zum dritten Weg einer Wirtschaftsordnung zwischen entartetem Liberalismus und Kollektivismus auf und im abschließenden Abschnitt 7.4 »Die deutsche Aufgabe im Abendland« bemüht er Röpke mehrmals als Kronzeugen für seine Gedanken.

7 Emil Brunner: Gerechtigkeit. Eine Lehre von den Grundgesetzen der Gesellschaftsordnung. Zürich 1943. Das Exemplar aus Baudissins Nachlass ist gestempelt: »Buchspende der Weltstudentenhilfe World Student Relief; 13, rue Calvin; Genf/Schweiz«. Das Exemplar in der Staatsbibliothek Hamburg ist gestempelt: »Schweizer Bücherhilfe für Kriegsgefangene. Swiss Book Donations for Prisoners of War «. Baudissins Arbeitsexemplar befindet sich ebenfalls in seinem Nachlass. Es kann nicht mehr festgestellt werden, welches der beiden Bücher Baudissin als erstes in Händen gehabt hatte; seine Anmerkung über den Fund von Röpke und dessen Verweis auf Brunner lassen jedoch vermuten, dass Baudissin Röpke zuerst gelesen hat. Zur Person Emil Brunners: Hans Heinrich Brunner: Mein Vater und sein Ältester. Emil Brunner in seiner Zeit. Zürich 1986.

8 Bedeutsam ist, dass mehr als die Hälfte der Nachträge in der Denkschrift Texte von Röpke und Brunner betreffen.

9 Kutz a.a.O.

10 Baudissin bemerkt häufig in seinen Briefen an Gräfin Dohna, wie wichtig für ihn bei seiner Gedankenarbeit der unmittelbare tiefergehende Gedankenaustausch mir ihr wäre. dem »Pastorenbrief « Nummer 114 vom 19. Oktober $1946^{11}$ an die Braut deutlich wird. Dort schreibt Baudissin u.a., dass die Zukunftsaufgabe einen Standpunkt zwischen zwei Polen fordere, »dass aus einer eindeutigen Beziehung gleich Verantwortung des Einzelnen zu Gott sich ebenso klare Beziehung zur Umwelt wie zur werten Eigenperson ergeben. Demut zu Gott gleich Liebe zu den Mitmenschen, Achtung vor ihren Leistungen und der Natur überhaupt bzw. das Empfinden, sich selbst zur Aufgabe gestellt zu sein «. Zum anderen sind mit den Bücherspenden aus der Schweiz nach Kriegsende wohl erstmals aktuelle Schriften im Gefangenenlager zu lesen gewesen und haben von daher wohl ganz generell für geistig neuen Wind gesorgt. ${ }^{12}$ Natürlich ist es auch das Thema des Buches: "Gerechtigkeit « selber, das Baudissin nicht nur aufgrund seiner Herkunft aus einem Juristen-Haus stets von sehr großer Bedeutung gewesen ist. Ebenso hat Brunners Ansatz, dekliniert über die verschiedenen sozialen Ordnungsebenen, Baudissin bei der Suche nach Neuorientierung für die generell zerstörte Wertewelt nahe gelegen. Es sind schließlich auch Brunners einzelne Aspekte und Fragestellungen, die von Baudissin zu dem Zeitpunkt bereits ähnlich gesehen und gedacht wurden, so dass hierüber sich ein sachlich-emotionaler Bezug leichter bilden und Baudissin den Weg zur Aufnahme des Neuen ebnen konnte.

Ein gutes Beispiel für diese thematische Nähe ist der nicht unbedeutende Gedanke der »Erschütterbarkeit« im Briefwechsel Baudissin - Dohna: Baudissin schreibt am 17.7.1943 im Zusammenhang mit der Suche nach Neuansätzen: »...ich bin ganz Deiner Ansicht, dass >Erschütterbarkeit< etwas sehr Positives - für den, der nicht erschüttert und nicht erschlagen wird, - sie ist für den wirkenden Menschen sogar eine Notwendigkeit. Ohne diese Möglichkeit ist eine Weiterentwicklung wie auch wesentlicher Einfluss auf die Umwelt nicht denkbar. Formung und Formen sind ja letztlich nur Aus- bzw. Umwertung solchen >Erschüttert-Gewesen-Seins`. Wer kein Organ für Derartiges hat, erhält keinen wirklich fördernden Ansporn und bleibt in sich stecken - das Über-Sich-Hinaus als erster Schritt bleibt ihm verwehrt. Allerdings sehe ich Dich verzeihend lächeln, wenn ich die unbegrenzte Erschütterbarkeit als zu weit gehende Forderung einschränken möchte. ... Gelegentliche Unerschütterbarkeit (nicht nur äußerlich, was nicht zur Debatte, ist doch notwendig, schon um zu sich bzw. zu etwas (Unterschied?) kommen zu können. ... Vielleicht versuchen wir es weiter mit erschütterbarer Erkenntnis, mit demütiger Offenheit den letzten Dingen glaubend, während man der übrigen - gleichgeordneten - Welt erkenntnismäßig zu Leibe geht. «13 Brunner seinerseits geht im Schlusskapitel von »Gerechtigkeit« der Frage nach, wie denn die vorher entworfenen »Folgerungen « praktisch werden könnten, denn es sei »ein weiter Weg von der Erkenntnis bis zur Verwirklichung des Gerechten ${ }^{14}$. Um die dabei bestehenden Hindernisse aus

11 Baudissin 2001 a.a.O. S. 156 ff. Dort wird auch deutlich, dass Baudissin während der Gefangenschaft darüber nachdachte, nach dem Krieg Theologe $\mathrm{zu}$ werden.

12 Über die Bedeutung dieser Büchersendungen äußert der Mitgefangene Globig sich an Baudissin in seinem Antwortbrief nach dem Lesen von »Ost oder West «.

13 Baudissin 2001 a.a.O. S. 65. Im Brief vom 6.4.1944 setzt Baudissin die Diskussion fort und spricht dort vom »richtigen Maß der Erschütterbarkeit «, ders.1994 a.a.O.

14 Brunner a.a.O. S. 309. 
dem Weg zu schaffen, brauche es »Erschütterungen der Seele, die nicht durch Erkenntnis, sondern nur durch >Buße und Glauben hervorgerufen werden können ${ }^{15}$.

\section{Spurensuche in Baudissins Exemplar von Brunners »Gerechtigkeit«}

In dem von Baudissin in der Gefangenschaft benutzen Exemplar von Brunners »Gerechtigkeit « befinden sich keine direkten Lesespuren wie Lesezeichen, Anstreichungen oder Bemerkungen. Was Baudissin von Brunner verarbeitet hat, ist daher in einem ersten Zugang nur über die Brunnerzitate in der Denkschrift zu erfahren. Dies geschieht an drei Stellen der Denkschrift:

Im 3. Kapitel »Deutschland und Russland«, Abschnitt 3.11 »Der Ostraum als Schauplatz der Heerzüge und Bildner des Preußentums «, merkt Baudissin zum Begriff »Preußentum«an, dass dies seit der Entwicklung des Luthertums zur Staatskirche durch die zunehmende Gleichsetzung von irdischer und göttlicher Autorität in sich eine Gefahr der »Mechanisierung « berge. Diesem hält er dann mit Luther - indirekt zitiert bei Brunner ${ }^{16}$ - den Gedanken des Widerstands entgegen: »Jedoch eine Widerstandspflicht mit dem Worte besteht immer, ein Widerstandsrecht mit Gewalt nur in besonders krassen Fällen, wenn eine tyrannische Obrigkeit Handlungen verlangt, die offensichtlich im Gegensatz zu Gottes Wort stehen." Dieses Verständnis von Widerstand ergebe sich aus dem »besonders fruchtbaren Spannungsverhältnis des Individuums Gott gegenüber«, das zunehmend seit Beginn des 19. Jahrhunderts in Preußen verloren gegangen sei.

Ein weiteres Mal nimmt Baudissin im Abschnitt $3.22 »$ Der soziale und staatliche Aufbau als asiatische Lösung « beim Thema »Individuum und Kollektivwesen « Brunner auf und zitiert ihn weitgehend wörtlich zu Geschichte und Äußerungsformen des Kollektivismus aus Brunners entsprechendem 11. Kapitel. ${ }^{17}$ Dabei geht es Baudissin besonders um die zweite von Brunner herausgearbeitete Form, den so genannten mechanischen Kollektivismus ${ }^{18}$, der nur vom $»$ radikalen Individualismus « her zu verstehen sei. Aufgrund der daraus sich entwickelnden »Atomisierung der Gesellschaft« und verbunden mit der Proletarisierung der Massen im liberalen Kapitalismus habe sich daraus der Kommunismus entwickelt.

Als Drittes fügt Baudissin gegen Schluss des Kapitels 5 »Auswertung der politischen Betrachtung « eine umfangreiche Anmerkung zur Frage der »Neuordnung der Welt und Europa « ein, in der er sich weitgehend auf Brunners 21. Kapitel »Die gerechte Völkerordnung « und dabei besonders auf die Abschnitte »1. Die Friedensordnung«, »2. Das Gesetz: Das

15 Dass. S. 311

16 Brunner a.a.O. Anm. 30. Dies wird von Brunner im Zusammenhang mit Souveränität und dabei dem Gedanken bemerkt, dass der Staat Gottes Ordnung sei und darum seine Grenzen am Gotteswillen habe. Das Thema Widerstand nimmt er dann noch einmal im 12. Kapitel in Zusammenhang von Gerechtigkeit und Naturrecht auf, s. dass. S. 110-112.

17 S. Brunner a.a.O. S. $94 \mathrm{ff}$.

18 Die beiden anderen Formen sind der organische sowie der universalistische Kollektivismus.
Völkerrecht « und »3. Das gerechte Völkerrecht« abstützt. ${ }^{19}$ Realisierbare Möglichkeiten einer »moralisch-praktischen Souveränitätsbegrenzung « aus dem Willen zum Frieden für die Völker und die Menschheit generell werden von Baudissin - ähnlich Brunner - noch nicht gesehen. Er sieht jedoch Möglichkeiten für eine Organisation der Völker im begrenzten Rahmen eines europäischen Zusammenschlusses; dabei sei es »vielleicht unser deutsches Schicksal, unsere äußere Einheit und Großmachtstellung auf dem Altar dieser europäischen Einigung zu opfern«. Diese Union fordert er geradezu als einzig mögliche Lösung für Europa: »Darüber hinaus gebietet gerade der Gesichtspunkt der Gerechtigkeit, d.h. die Forderung der Stabilität, Formung einer solchen Union«, da andere Denkansätze über des »Selbstbestimmungsrecht der Völker « oder über »die historische Ableitung von Ansprüchen« von »voll souveränen Staatsgebilden « wieder nur »neue oder alte Ungerechtigkeiten aufrichten muss «.

Sucht man weiter nach indirekten Lesespuren, so fällt beim Durchblättern von Baudissins Arbeitsexemplar der Brunnerschen »Gerechtigkeit « auf, dass aus dem ersten Teil »Grundlagen « nur wenige Stellen sich von selber öffnen. Dies sind:

Deckblatt

Vorwort

Inhaltsverzeichnis

Seiten 30/1 zu »Gerechtigkeit und Gleichheit «

Seiten 62/3 zu »Das Göttliche Gesetz der Gerechtigkeit»

Seiten 114/5 zu »Die geschichtliche Relativität« in »Statische und dynamische Gerechtigkeit « sowie

Seiten 144-147 zu »Die zehn Gebote« in »Die Gerechtigkeit und die biblische Offenbarung « mit Übergang zu »Gerechtigkeit und Liebe«.

Im Gegensatz dazu schlagen sich im zweiten Teil »Folgerungen« wesentlich mehr Seiten auf:

Seiten 158/9 zu »Der gerechte Aufbau der Ordnungen«

Seiten 210/1 zu »Die gerechte Ordnung der Wirtschaft « in »Kapitalismus und Kommunismus «

Seiten 222/3 zu »Der Massenmensch und die gerechte Gesellschaftsordnung «

Seiten 240-243 zu »Ungerechtigkeit des totalen Staates«

Seiten 252-257 zu »Gerechte Macht « in »Die gerechte Ordnung des Staates«

Seiten 268-271 zu »Die gerechte Völkerordnung «

Seiten 272/3 zu »Die Friedensordnung « in »Die gerechte Völkerordnung «

Seiten 280/1 zu »Die gerechte Völkerordnung«

Seiten 288-291 zu »Das gerechte Völkerrecht«

Seiten 306/7 zu »Schluss: Grenzen« sowie

Seiten 318-321 Anmerkungen 27-34 zu Kapitel 10-12 aus Teil I.

19 Vrgl. Brunner a.a.O. S. 268-297. 
Bei aller Behutsamkeit der Interpretation dieser Befunde wird deutlich, dass Baudissin die »Folgerungen « besonders interessiert haben. Dabei ging es ihm eindeutig um die Fragen der gerechten Völkerordnung mit der nach einer Friedensordnung sowie des gerechten Völkerrechts. Dies stimmt mit seiner ausgiebigen Anmerkung zum Thema Friedensordnung in Kapitel 5 der Denkschrift überein.

Ebenso haben ihn anscheinend die Fragen nach der Staatsordnung und dabei nach der Ungerechtigkeit des totalen Staates stärker beschäftigt, was zum einen im Zusammenhang mit seinen Zitaten in Abschnitt 3.22 der Denkschrift über den Kollektivismus stehen könnte. Zum anderen passen dazu die Spuren bei der Anmerkung 30, da dort die von Baudissin in Abschnitt 3.11 der Denkschrift zitierte Stelle über das Widerstandsrecht nach Luther zu finden ist.

Anhand der Anmerkung 30 wird zusätzlich deutlich, dass Baudissin den I. Teil nicht nur überflogen haben kann, da ihn das Thema Widerstand aus sehr persönlichen Gründen stark betroffen hatte ${ }^{20}$, diese Fundstelle bei Brunner sich aber nicht einfach logisch erschließt.

Der Leseschwerpunkt beim 14. Kapitel über das Thema Gerechtigkeit in der biblischen Offenbarung und besonders im Dekalog sowie beim Kapitel 15 über »Gerechtigkeit« und Liebe entspricht zum einen Baudissins christlicher Grundhaltung und wirft zum anderen ein Licht auf die Bedeutung von Äußerungen wie »Liebe« und »bloß ein guter Christ sein wollen«, die er für dritte häufig unverständlich oder zumindest erstaunlicherweise als letzte Antworten für praktische Lösungen bei Sachfragen immer wieder bis an sein Lebensende gemacht hat. $^{21}$

Die Spur in den Anmerkungen deutet schließlich auch auf Baudissins grundsätzlich rechts-orientiertes Denken hin, da sich dort die zweieinhalbseitige Anmerkung 34 über die Diskussion des Naturrechts aus Sicht der theologischen sowie juristischen Disziplinen befindet.

In umgekehrtem Sinne ist auch festzuhalten, dass Baudissin die Seiten 225 bis 227 wohl nicht mit besonderem Schwerpunkt gelesen hat, in denen Brunner sich eingehend mit der Ordnung der Armee beschäftigt und sie modellhaft als ein »Beispiel echter Ordnung « herausstellt. Daraus ist zu schließen, dass Baudissin sich 1946 nicht vordergründig mit Fragen aus seinem Berufsfeld, nämlich der Reform von Streitkräften, beschäftigt hatte, sondern dass er mit der Denkschrift zur "Schicksalsfrage « wirklich auf der Suche nach genereller Neuorientierung, nach einem geistig-ethischen Neuanfang für sich, die Gesellschaft in Deutschland und Europa, wenn nicht gar

20 Dazu s. Claus von Rosen: Frieden - Widerstand - Demokratie. Geistige und sittliche Gründe in Baudissins Konzeption Innere Führung. In: Martin Kutz (Hrsg.): Gesellschaft, Militär, Krieg und Frieden im Denken von Wolf Graf von Baudissin. Band 23 der Reihe: Forum Innere Führung, Baden-Baden 2004, S. 25-44.

21 Vrgl. Claus von Rosen: Wolf Graf v. Baudissin zum 75. Geburtstag. In: Wolf Graf von Baudissin: Nie wieder Sieg! Programmatische Schriften 1951-1981. München 1982, S. 7 ff. Siehe auch Rosen, Claus v.: Geistige Grundlagen in Werk und Wirken von Wolf Graf v. Baudissin. In: Führungsakademie der Bundeswehr (Hrsg.): Jahresschrift 1994/1995. Hamburg 1995, S. 49 ff. der gesamten Welt war. ${ }^{22}$ Dennoch darf hier der Gleichgang von Gedanken im weiten Bereich bei Brunner und Baudissin nicht übergangen werden, der sich in Baudissins Denken generell finden lässt, besonders auch festzumachen an seiner Gesamtkonzeption der Inneren Führung, der aber sich in dieser Denkschrift aufgrund ihrer Themenstellung nur sehr bedingt erschließt. ${ }^{23}$

Auf die leitende Frage ist hier als erste Antwort festzuhalten: Aus diesen Spuren tritt ganz besonders die der Suche nach einer Friedensordnung hervor. Dieses Thema hat Baudissin sich erst mit der Lektüre von Brunners »Gerechtigkeit « erschlossen. Frieden ist für Baudissin seitdem bis an sein Lebensende das bestimmende Thema für sein gesamtes Denken und Handeln gewesen ist: Bald nach der Entlassung aus der Gefangenschaft trat Baudissin verschiedentlich in der kirchlichen Arbeit mit Vorträgen zum Thema Frieden auf. ${ }^{24}$ Die Arbeiten zur Konzeption Innere Führung beim Aufbau der Bundeswehr hatten als einen der - um nicht zu sagen: den - zentralen ethischen Eckpunkte ebenfalls das Thema Frieden. ${ }^{25}$ Baudissins von Flexibilität bestimmten militärstrategischen sowie politischstrategischen Vorstellungen als Stellvertretender NATO-Befehlshaber in der Zeit der Massiven-Vergeltungs-Strategie und bei der Entwicklung der Strategie der flexible response waren davon gekennzeichnet, Instrumente zur Bewahrung und Wiederherstellung des Friedens zu entwickeln. ${ }^{26}$ Und nach seinem Ausscheiden aus dem militärischen Dienst befasste Baudissin sich in seinen letzten 25 Lebensjahren als Forscher und Hochschullehrer mit friedensrelevanten Fragen zur sicherheitspolitischen Beratung der Politik.

\section{Frieden in Baudissins Denkschrift »Ost oder West« von 1947}

Baudissin beginnt seine Anmerkung ${ }^{27}$ in der Zusammenfassung der Denkschrift mit einem Zitat aus Brunner: »Die Konzentration der Machtmittel im Staat, vor allem die Monopolisierung des Tötungsrechtes, war zwar notwendige Voraussetzung zur Überwindung anarchischer Zustände, des bellum omnium contra omnes zwischen den Individuen, ist aber nunmehr im zwischenstaatlichen Bereich der entscheidende Faktor internationaler Anarchie - »der nach innen segensreich-

22 Beredtes Zeugnis dieser generellen Neuorientierung ist Baudissins Briefwechsel mit seiner Braut - s. Anmerkung 2. Vergl, auch: Gespräch mit Wolf Graf von Baudissin. In: Axel Eggebrecht (Hrsg.): Die zornigen alten Männer. Gedanken über Deutschland seit 1945. Hamburg 1979, S. 203 ff, besonders S $207 \mathrm{f}$. und $211 \mathrm{ff}$.

23 Hier geht es u.a. um Fragen zum Neuanfang aus einer ethischen Neuorientierung allgemein und in der Art einer Renaissance im speziellen, zum Primat des Individuums, zum Individualismus gegenüber Kollektivismus, zur gerechten Ordnung gegenüber dem Totalitären, zum Föderalismus, zur Demokratie, zur Ordnung und Gerechtigkeit in Gesellschaft, Staat und dessen Gliederungen, zur Ungleichheit und deren strukturbildender Bedeutung allgemein wie auch speziell in der Arbeitswelt und dem Militär sowie zur Staatsbürgerlichen Erziehung.

24 S. Rosen 1982 a.a.O. S. 13 ff. So ist auch in der Bibliographie von 1982 als erstes schriftliches Dokument Baudissins Vortrag von 1950/51 über »Frieden auf Erden?« aufgeführt, dazu s.u.

25 Vrgl. Rosen 2004.

26 Dies ist das Ergebnis der Lehrgangsarbeit vom Major Renk an der Führungsakademie der Bundeswehr: Baudissins Wirken als General bei AFCENT und SHAPE. Hamburg 1998. Vrgl. a. Axel F. Gablik: Strategische Planungen in der Bundesrepublik Deutschland 1955-1967: Politische Kontrolle oder militärische Notwendigkeit? Baden-Baden 1996, besonders S. $299 \mathrm{ff}$

27 Baudissin 1947, Anm. zu Kap. 5. S.a. Rosen 1995b, S. 53 ff. 
machtvolle Staat wird nach außen zum brutalen Vergewaltiger und Räuber«. Die Beziehungen der Völker werden praktisch nur vom staatlich nationalen Egoismus bestimmt, d.h. jeder Staat nimmt soviel an Macht und irdischen Gütern, als irgend möglich, und gibt nur ab, wenn und was unvermeidlich.«28

Brunner indirekt zitierend, geht Baudissin dann weiter aus von der Ablehnung der moralisierenden Unterscheidungskriterien »friedliebend « und »kriegerisch « für Schuld und Unschuld am Krieg und kommt zu der Feststellung, dass die entscheidende Dominante der zwischenstaatlichen Beziehungen der nationale und imperiale Machtegoismus in zwei Formen sei, der konservativ-unkriegerische und der aggressiv-kriegerische. Angesichts der 1945 augenscheinlichen »Existenzfrage « der abendländischen Zivilisation oder gar der Menschheit überhaupt müsse »mit allem Ernst und guten Willen an die Einrichtung einer Völkerordnung gegangen werden $\aleph^{29}$. Brunner folgend, sucht er nun, »analog der innerstaatlichen Entwicklung, eine einigermaßen gerechte Verteilung der Pflichten und Rechte der einzelnen Staaten zu finden, wie einen Weg zur laufenden Anpassung und Organisation an die unaufhaltsame Weiterentwicklung bzw. Mittel zur Verhinderung anarchischer Tendenzen «.

Die drei im Weiteren nach Brunner dargestellten Möglichkeiten einer Friedensordnung - durch zwingende Macht in Form der Pax Romana, durch völkerrechtlichen Friedensvertrag wie durch föderalistisch-kooperative Bildung einer überstaatlichen Macht $^{30}$ - bieten auch für Baudissin keine »institutionelle Lösung des Anarchieproblems « zwischen den Völkern. Dies zu erkennen sei die Pflicht jedes Menschen, der verantwortungsvoll am Problem des Völkerfriedens zu arbeiten habe und sich nicht mit utopischen Phantasien zufrieden gebe. ${ }^{31}$

Dem dritten Modell scheint Baudissin jedoch eine gewisse Chance der Realisierung zuzubilligen, wenn er weiter, auf Brunner verweisend, ausführt, dass die einzige Friedensgarantie der Zukunft darin liege, »den jetzt maximalen Friedenswillen aller « zu nutzen, »der Friedenswille der Staaten und Völker, der als solcher auch der Wille sein muss, für gerechte Ansprüche anderer nationale Opfer zu bringen $\ll^{32}$. Dazu sei »von innen her auf die Menschheit einzuwirken, um des Friedens, d.h. der eigenen Existenz willen auf eine rein egoistische Machtpolitik zu verzichten und im Interesse dieses Friedens, gewisse minimale Forderungen völkerrechtlicher Gerechtigkeit und Friedlichkeit zu er füllen, auch wenn sie Opfer kosten «. Wie Brunner hofft Baudissin anscheinend, dass aus einer solchen »moralisch-praktischen Souveränitätsbegrenzung « sich gewisse Institutionen überstaatlicher Zusammenarbeit ergeben, die dann mit der Dauer ihres Bestehens immer mehr Autorität erlangen. »Zwar kann kein Schiedsgericht eine

28 Vrgl. Brunner a.a.O. S. 273

29 Vrgl. Brunner a.a.O. S. 275

30 Vrgl. Brunner a.a.O. S. 277 ff. Die dritte Möglichkeit, Friede durch das mit Macht verbundene Recht, scheint für Baudissin am ehesten erfolgversprechend zu sein. Nach Brunner geht es dabei um die »föderalistisch-kooperative Bildung einer überstaatlichen Macht, die den Frieden gewährleistet indem sie, nach dem gemeinsamen Beschluss der Kooperanten, den einzelnen Friedensstörer, das heißt den, der sich dem Beschluß der Völkerunion nicht beugt, zum Gehorsam zwingt«. Vergl. Brunner a.a.O. S. $280 \mathrm{ff}$.

31 Vrgl. Brunner a.a.O. S. 282.

32 Vrgl. Brunner a.a.O. S. 283 f. Bei Brunner sind die Worte >Friedenswille< und ssolcher< gesperrt gedruckt. Dies weist darauf hin, welchen Stellenwert er dem Friedenswillen gegenüber institutionellen Möglichkeiten gibt.
Großmacht in der Ausführung ihres Willens hindern, sondern nur die Erkenntnis, dass ein machtegoistisches Benehmen den Keim eines nächsten Weltkrieges in sich birgt. «33

Baudissin referiert bis hierher Brunner, ohne dabei dessen analytische Gliederung der Gedanken zur »gerechten Völkerordnung « aufzunehmen: Brunner entwickelt seine Ausführungen in diesem Kapitel als Antwort auf anarchische Zustände von Gewalt anhand eines Stufenmodells im Hinblick auf die Realisierung von Gerechtigkeit im Verkehr zwischen den Völkern ${ }^{34}$ : 1. Die Friedensordnung 2. Das Gesetz: Das Völkerrecht 3. Das gerechte Völkerrecht 4. Die gerechte Machtverteilung 5. Die außer- und überpolitischen Möglichkeiten. Dabei ist entscheidend, dass Brunner keiner der fünf Stufen-Möglichkeiten eine Chance zur Garantie des Friedens zumisst, wenn sie nicht vom Friedenswillen der Beteiligten getragen werde. Brunner betont sogar, »dass in Zukunft der Wille zum Frieden die Dominante aller zwischenstaatlichen Politik sein muss «35. Baudissin übergeht nicht nur dieses Muss bei Brunner, sondern überhaupt dessen Auffassung, dass auf allen Stufen und bei jedem der drei Modelle der ersten Stufe zu einer Friedensordnung der Friedenswille konstitutiv für die Verwirklichung von Frieden sei. ${ }^{36}$

Brunner fasst im Schlussgedanken über »Grenzen « der theologischen Gedanken seinerseits Überlegungen für die Realisierung von Frieden in der Welt zusammen, die jedoch vermutlich nur bedingt Antworten auf Baudissins politisch-praktische Fragen zu jener Zeit liefern konnten. Brunner spricht über die Wiederentdeckung der »Willensziele « und deren Hervorhebung vor den Mitteln, weiter über die Hoffnung auf den »uneigennützigen Willen zur Gerechtigkeit« wenigstens einer Minderheit von Parteigängern, über »staatsbürgerliche Erziehung « zum Gerechtigkeitssinn als »Geschehnis der >Wiedergeburt « ü über das »Neuwerden und Anderswerden«, über einen Willen zur Tat aufgrund einer »Erschütterung der Seele, die nicht durch Erkenntnis, sondern nur durch >Buße und Glaube hervorgerufen werden können « sowie über Liebe, »ist doch Gerechtigkeit nichts anderes als die Gestalt der Liebe, die in der Welt der Ordnungen und Institutionen Kurs hat « ${ }^{37}$. All diese Gedanken haben aber dennoch Platz in denen von Baudissin gehabt, wie seine späteren Aussagen über Frieden zeigen werden.

Im Gegensatz zu Brunner sieht Baudissin jedoch in seiner Denkschrift - wie bereits gesagt - gewisse Möglichkeiten zur praktischen Verwirklichung einer Friedensordnung der Völker

33 Vrgl. Brunner a.a.O. S. 283-287.

34 Die dabei benutzten Kriterien hat Brunner bereits bei der Darstellung der "gerechten Ordnung des Staates« verwendet, vrgl. ders. a.a.O. S. $233 \mathrm{ff}$.

35 Vrgl. Brunner a.a.O. S. 272 und S. 296.

36 So resümiert Brunner bereits im einleitenden Abschnitt zu Kapitel 21, dass neben unbestechlichem Wirklichkeitssinn der unbedingte Wille zum Gerechten den christlichen Staatsmann ausmache - vrgl. ders. a.a.O. S. $271 \mathrm{f}$. Und im vierten Abschnitt betont er, dass ausschließlich der »einsichtige Friedenswille der Großmächte« eine Garantie böte. Dazu sei »die Mitsprache aller Völker allerdings notwendig «. Dass. S. 298. Baudissins Zitate stammen mit Masse aus den beiden ersten Abschnitten zur »Friedensordnung « mit den drei Modellen sowie zum »Völkerrecht« (allgemein); die Zitate zum Friedenswillen stammen zum einen aus Brunners erstem Abschnitt zur »Friedensordnung « und zum anderen aus dem dritten zum "gerechten Völkerrecht $\ll$.

37 Brunner a.a.O. S. 307-312. Auch der kurz davor von Brunner geäußerte Gedanke, dass »Gerechtigkeit ohne Macht ohnmächtig « sei, wird von Baudissin hier übergangen - trotz entsprechender Überlegungen vorher. 
»in wirkungsvoller Form ${ }^{38}$ für einen Zusammenschluss Europas. Er verfolgt somit einen ganz anderen Ansatz als Brunner. Er hat damit das Thema Frieden insgesamt für sich - neu - entdeckt und ihm für sein weiteres Denken und Wirken entscheidende Bedeutung und Richtung gegeben: Er ist sehr viel stärker auf die politische Praxis bezogen; seine Gedanken kreisen eindeutig um die Frage der Realisierung von Frieden. Dennoch: Brunners Gedanken und Ideen zu Frieden sind für Baudissin dabei nicht nur anregend neu, sondern wirken auf ihn sogar revolutionär. Einzelne Aspekte sammelt er daraus eklektisch wie aus einem Steinbruch auf und arbeitet sie in seine Denkschrift ein.

\section{Frieden in Baudissins Vorträgen im Rahmen der Evangelischen Kirche 1950/1951}

In Vorträgen im kirchlichen Bereich Anfang der 1950er Jahre betont Baudissin eine individuelle christliche Sichtweise für die Frage nach »Frieden auf Erden $\ll^{39}$ - wie er sein Thema nannte. Auch wenn Baudissin in diesem Vortragsmanuskript an keiner Stelle Brunner namentlich erwähnt, so ist doch deutlich zu erkennen, dass er bei Erstellung des Manuskriptes sich noch sehr genau an die Lektüre von »Gerechtigkeit « knapp vier Jahre vorher erinnert hatte. Manche Begriffe oder Wendungen wie »Friedliebende «, "gerechter Frieden«, »gerechte Ordnung $«$, »Freiheit und Ordnung in Gerechtigkeit «, die Verneinung von Rezepten für die Schaffung des Friedens auf Erden aus dem biblischen, »Opferbereitschaft «, »suum cuique«, »gemeinsame Existenzbedrohung « oder dem Friedensstörer das »Wasser abgraben« weisen unmittelbar auf Brunner hin.

Baudissin geht von einer »negativen « Bestimmung von Frieden aus als Abwesenheit von Gewalt und Spannungen und bezeichnet dies als eine »Illusion«. Dann beschreibt er Frieden "positiv« als eine Sehnsucht der Menschheit und definiert ihn als allgemein menschliches, individuelles Problem: »Frieden ist gegeben, wenn $<$ das $>$ Ich gewillt $<$ ist $>$, alle Spannungen ohne Gewalt zu überwinden (Opferbereitschaft), Polarität zu bejahen (Individualität), und im Vertrauen, dass < das $>$ Du gleichen Willens <ist $>$ d.h. wenn alle Partner bestimmte Ordnungsprinzipien anerkennen. <Dies ist aber $>$ nur möglich, wenn jeder den Nächsten und seine Sphäre genau so achtet wie <die> eigene und das Anderssein des anderen als fruchtbar bejaht - suum cuique - achte Deinen Nächsten wie Dich selbst.« Dazu müsse man sich bewusst sein, dass Gewalt entstehe, »wenn Spannungen nicht ertragen werden«. Kampf dürfe aber nicht gegeneinander, sondern nur »nebeneinander um des Besseren Willen« - geführt werden.

Damit betont Baudissin den inneren Frieden, den »Friede mit sich selbst«, vor dem politischen Frieden. Jener sei »nur aus dem freien Entschluss und der Verantwortung von opferbereiten, vertrauensvollen Menschen zu schließen«; der politische Frieden könne aber »nur in uns selbst beginnen «. Er appelliert an die »Verantwortung der Friedliebenden zum Frieden-Stiften«, d.h. zur »Arbeit an sich selbst, um Vertrauen

38 Vrgl. Brunner a.a.O. S. $280 \mathrm{f}$.

39 Baudissin 51,1. S.a. Rosen 1982, S 13f. - Aus dem handschriftlichen Stichwortmanuskript wird der folgende Lauftext rekonstruiert. zu erringen und zu stärken « sowie zur »Schaffung und Aufrechterhaltung <einer> gerechten Ordnung (Augustin), die von den meisten anerkannt « wird. Dabei sieht er aber auch das Problem »vorsätzlicher Friedens-Störer «, denen man als »Hauptweg « - das »Wasser abgraben «, dabei »aber auch < das> Schwert als Damm (Vakuum) einsetzen « müsse; der andere Weg sei der »echter Intoleranz (Wahrheit und Ordnung) als Vertrauenshilfe«, der dritte Weg heißt »Vergeben, Nachgeben und Aufgeben « und der vierte bedeute »Übertragung < der Aufgaben $>$ an <den $>$ Staat - doch Notwehr - Notstand - ohne Rache zur Wiederherstellung < des> status quo «.

Bei der nächsten Frage nach Aussagen der Bibel zu Krieg und Frieden benutzt Baudissin zu weiten Teilen dieselben Bibelstellen wie Brunner und unterscheidet - Brunners Gedankengang z. B. zum Verhältnis von Liebe und Gerechtigkeit folgend - nach dem biblischen Frieden und Frieden auf Erden. Der biblische sei nur endzeitlich und biete kein »Rezept« zur Verwirklichung des Friedens auf Erden. ${ }^{40}$

Baudissin fragt nun angesichts der "gemeinsamen Existenzbedrohung « weiter nach möglichen Konsequenzen. Wie Brunner verwirft Baudissin die Gedankenmodelle des Heiligen sowie des Gerechten Krieges. Er ist davon überzeugt, dass es eine »Tatsache <sei>: Keine noch so hohe Frage <ist ein> Kriegsgrund <sondern bringt> nur neues Unrecht und Leid «. Daraus folgert er, dass Frieden »doch nur aus freiem Entschluss und Verantwortung von opferbereiten verantwortungsvollen Menschen« möglich sei. Und, auf die politische Ebene zielend, spricht er von »neuem gelenkten Gebrauch der Macht«, von einer »neuen Aufklärung«, die die Grenzen des Menschen deutlich mache - wie bereits von den Atomphysikern beschritten - sowie von »sozialer Gerechtigkeit in Demokratie«. Dazu empfiehlt er, den »Blick vom Fernziel zum Nahziel«, auf das »politisch Konkrete« zu richten, »Gemeinsames und Anknüpfungspunkte« der einander gegenüberstehenden Systeme zu suchen, um »Möglichkeiten <aufzutun>, Zustände zu bessern«.

Nach Baudissins Verständnis von Frieden haben Christen ein tragisches Verhältnis zur Welt: Frieden bedeute für ihn »stets Leiden«. Dabei sei es eine für Christen typische Aufgabe, das »Durcheinander zu ordnen <und> falsche Gegensätze ... zu überbrücken, längst überholte Fronten zu durchbrechen, Freiheit und Ordnung in Gerechtigkeit zu vereinen, <das> Bild $<$ vom> Christlichen Abendland ... zu leben «. Der Beitrag der Christen - als Kirche, Gemeinde wie Einzelner - zum »Frieden des Geistes und des Gewissens « sei es, allgemein gesprochen, »aus dem Anti zum friedlichen Pro« zu kommen. Konkreter bedeute das, den Frieden zu stabilisieren durch:

40 In diesem Zusammenhang ist auf Baudissins handschriftliches Vortragsmanuskript »Christ und Wehrdienst «, ders. 52,6, hinzuweisen, in dem er sagt, dass die »Bibel keine Rezepte für Frieden auf Erden « liefere, »sondern Raum für ernste Selbstprüfung + Entscheidung im konkreten Fall. Klar, dass die Kinder Gottes Friedensstifter sein sollen, nicht generell gesagt, wie Verhalten in friedloser Zeit «. - Baudissins Schriften von hieran zitiert anhand de Zählung in der Bibliographie in: Wolf Graf von Baudissin: Nie wieder Sieg! Programmatische Schriften 1951 - 1981. Hrsg. von Cornelia Bührle und Claus von Rosen, München 1982, S. 272-312. Die ersten beiden Ziffern stehen für das Entstehungsjahr, die folgenden für die laufende Nummer. 
- Verhandlungsbereitschaft,

- Aufhebung der Angst im Wissen um eine letzte, nicht irdische Instanz,

- Aufhebung der Vergeltungskette im Wissen um die eigene Schuld und die Bedürftigkeit der Vergebung sowie

- Mut zum Wagnis neuer Wege aus dem Wissen um die Unzulänglichkeit der bisherigen und um die eigene Unabhängigkeit vom Erfolg sowie

- Gebet.

Enthalten der zweite, dritte und fünfte Punkt eine scheinbar ausschließlich menschlich-individuelle Komponente mit stark transzendentaler Implikation, so wird anhand des ersten und vierten in Verbindung mit den politisch-allgemeineren Bemerkungen vorher deutlich, dass Baudissin hiermit - wie schon in seiner Denkschrift von 1947 angedeutet - ganz handfeste politisch-praktische Forderungen und Institutionen im Auge hat.

Baudissins eigener, auf praktische Politik zielender Ansatz wird in den Vorträgen dieser Zeit weiter konkretisiert: Der Gedanke an Stabilisierung des gerade bestehenden Friedens als Nahziel, die Betonung von Verhandlungsbereitschaft, die Frage nach Möglichkeit zur Wiederherstellung des Status quo nach Störung des Friedens, aber auch die individuellen, deutlich christlich bestimmten Gedanken zum Frieden sind eindeutig praktisch. Die duale Betrachtung von Frieden kennzeichnet dabei über die Praxisorientierung hinaus Baudissins Ansatz: Die individuellen Aspekte von Frieden werden von Baudissin in das Politikfeld eingebracht. Dies wird besonders an den Ausführungen zur Überwindung - so verstanden falscher Gegensätze zwischen den Systemen und dem Aufruf zum Mut zum Wagnis neuer Wege aus dem Wissen um die Unzulänglichkeit deutlich. Seine Auffassung vom tragischen Verhältnis des Christen zum Frieden beinhaltet, dass für Baudissin Frieden niemals als konfliktfreier >friedlicher $<$ Zustand zu denken ist. Und dennoch - das ist hier bereits festgestellt - gibt es für Baudissin zu Frieden auf Erden als politischem Zustand keine Alternative. Beides hat auch für seine Grundgedanken zur Konzeption Innere Führung der neu aufzustellenden Streitkräfte in der Bundesrepublik Deutschland wesentliche Bedeutung.

\section{Frieden in Baudissins Überlegungen zur Inne- ren Führung und Strategie 1951-1968}

Die Bedeutung, die Baudissin Frieden bei seinen Arbeiten zum Inneren Gefüge und der Konzeption Innere Führung für die Bundeswehr beimaß, ist nicht unmittelbar aus den Themen seiner Beiträge aus den Jahren seines aktiven Dienstes im Amt Blank, im Verteidigungsministerium, als Brigadekommandeur sowie in seinen Verwendungen in NATO-Stäben und dem NATO-Defence-College zu entnehmen. Wo es um die von ihm so genannten geistig-sittlichen Grundlagen des Soldaten der Bundeswehr geht, um dessen ethisches Fundament, sowie um die Frage nach der politischen Legitimation von Streitkräften, nach deren politischem Zweck, nach dem Kriegsbild und Stra- tegie im Atomzeitalter, dort greift Baudissin immer wieder an zentraler Stelle auf Frieden als Letztbegründung zurück. ${ }^{41}$

In seiner ersten öffentlichen Äußerung als Mitarbeiter des Amtes Blank im Dezember 1951 an der Evangelischen Akademie in Hermannsburg ${ }^{42}$ formulierte Baudissin, was wie ein Paukenschlag gewirkt haben muss: Für künftige Streitkräfte sei Frieden ${ }^{43}$ das Ziel und Abschreckung ${ }^{44}$ dazu das politische Mittel. »Wir haben ernsthaft und redlich umzudenken und uns bewusst zu machen, dass der Soldat in aller erster Linie für die Erhaltung des Friedens eintreten soll; denn im Zeitalter des absoluten Krieges mit seinen eigengesetzlichen, alles vernichtenden Kräften gibt es kein politisches Ziel, welches mit kriegerischen Mitteln angestrebt werden darf und kann, außer der Verteidigung gegen einen das Leben und die Freiheit zerstörenden Angriff.«

Und weiter heißt es in dem Vortrag ganz praktisch - bis hin zu Gedanken für die Entwicklung eines positiven Friedens in der eigenen Gesellschaft und in Europa: »Die Streitkräfte können Wesentliches zur Stärkung und Wahrung des Friedens beitragen, indem sie

- durch ihre militärische Abwehrstärke dem Gegner die Versuchung eines risikolosen Angriffs nehmen;

- durch die Existenz das Klima der Angst abbauen helfen und so die Menschen aus der Reaktion zur Aktion freimachen;

- durch vielfältige menschliche Begegnung und sachliche Zusammenarbeit das europäische Gemeinschaftsbewusstsein verbreitern und vertiefen;

- die Jugend an den Staat heranführen;

- den Wurzellosen einen Zugang zum späteren Zivilberuf öffnen;

- durch ehrliche Bemühungen um den Menschen und ein Leben in neuen Formen der fortschreitenden Funktionalisierung und Enthumanisierung einen Damm entgegenstellen.«

In einem Vortrag in Bad Boll von 1952 fasste er die politischen und individuellen Aspekte von Frieden - noch für die Streitkräfte der Europäischen Verteidigungsgemeinschaft - zusammen: »Dieser Soldat der E.V.G. soll sichtbar dem Frieden in

41 Dies hatte dazu geführt, dass sein Mitarbeiter Dr. Will 1953 bei den Vorarbeiten zum Soldatengesetz - vergeblich - forderte, in der Präambel die »Pflicht zur Friedenswahrung nach innen und außen« aufzunehmen. S. Tagebuch der Gruppe Inneres Gefüge am 29.9. und 1.10.1953.

42 Baudissin 51,5; ders.: Soldat für den Frieden. Entwürfe für eine zeitgemäße Bundeswehr. Hrsg. von Peter v. Schubert. München 1969, S 23 ff. S.a. Detlef Bald: Graf Baudissin und die Reform des deutschen Militärs. In: Hilmar Linnenkamp/Dieter S. Lutz (Hrsg.): Innere Führung. Zum Gedenken an Wolf Graf von Baudissin. Reihe: Demokratie, Sicherheit, Frieden Band 94, Baden-Baden 1995 , S. $19-53$, hier: S. 38ff.

43 Im Vortrag vor dem Einweisungslehrgang für höhere Stabsoffiziere und Generale 1956 in Sonthofen führte Baudissin entsprechend aus: »Vom Frieden her bekommt die Kriegführung ihren Auftrag und ihre Grenzen.« ders. 56,1. S.a. Bundesministerium für Verteidigung, Führungsstab der Bundeswehr I 6 (Hrsg.): Handbuch Innere Führung. Hilfen zur Klärung der Begriffe. Bonn 1957a, S. 59 sowie Bundesministerium für Verteidigung: ZDv 11/1. Leitsätze für die Erziehung des Soldaten. Bonn 1957b, die unter seiner Leitung entstanden ist. Dort heißt es in Ziffer 1 programmatisch: »Die Bundeswehr schützt Frieden und Freiheit des deutschen Volkes. Sie sichert gemeinschaftlich mit den Soldaten der freien Welt die auf Recht begründeten Lebensordnungen, die der europäische Geist seit Jahrhunderten formt.«

44 In einem Brief an Herrn Pansius-Heide vom 13.6.1952 schreibt Baudissin als Begründung für seinen Entschluss, »doch« ins Amt Blank gegangen zu sein: »Da ich aber zu der Erkenntnis kam, dass der Frieden nur über eine Verteidigungsbereitschaft zu bewahren ist, glaubte ich, mich der praktischen Konsequenz dieser Auffassung nicht entziehen zu dürfen.« 
Freiheit dienen in dem Bewusstsein, dass im Zeitalter des autonomen ${ }^{45}$ Krieges kein politisches Ziel so wichtig sein kann, als dass es mit kriegerischen Mitteln angestrebt werden darf. Er muss wissen, dass die beste Friedenssicherung in seiner Kraft und Bereitschaft zur Abwehr liegt, aber auch davon überzeugt sein, dass er für eine trotz aller Gebrechlichkeit verteidigungswerte Ordnung und ganz konkret für seinen Nächsten steht. Dieses kann der Soldat nur, wenn er im menschlichen Frieden zu seinen Vorgesetzten, Kameraden und Untergebenen lebt. Dafür soll das >Innere Gefüge' Sorge tragen. Aber darüber hinaus muss sich der Soldat des deutschen Kontingents auch im Frieden mit seinem Volke fühlen, aus dem er nicht auszusondern ist. Somit richtet sich die Frage nach dem Inneren Gefüge nicht nur an den zukünftigen Soldaten und seine nächsten Angehörigen, sondern an alle verantwortlich denkenden Menschen. $« 46$

Die individuelle Komponente von Frieden wird von Baudissin auch in den folgenden Jahren weiter betont. So sagte er 1953 bei einer Tagung zur Vorbereitung der »Leitsätze für Erziehung «: »Der Zustand den wir heute Frieden nennen, scheint alles andere als friedlich zu sein. Der permanente Bürgerkrieg ohne räumliche und zeitliche Grenzen und ohne Beschränkung der Kampfmittel stellt uns vor schwerwiegende Probleme, die wir gerade im soldatischen Raum als etwas wirklich Neues nicht übersehen dürfen. Denn die Grenze zwischen den Lagern, die Grenze zwischen den beiden Lebensordnungen läuft tatsächlich in den Herzen des Einzelnen, also auch durch die Herzen der Soldaten. «47 Entsprechend formulierte er bei seinem Abschied aus der Unterabteilung des Ministeriums 1958 ein »Erziehungsziel zum Freiseinsollen«: »Doch von den Freien allein hängt es ab, ob wir die Lage bewältigen und ob wir eine Ordnung schaffen werden, die unter den veränderten Bedingungen ein Höchstmaß an Freiheit, Recht und Menschenwürde bietet. Das wäre der entscheidende Beitrag zum Frieden der Welt. « ${ }^{48}$

Und zu Ende seiner militärischen Dienstzeit stellte Baudissin im Vortrag »Beitrag des Soldaten zum Dienst am Frieden« fest: »Auch der Soldat hat seinen Beitrag zum Frieden zu leisten, wenngleich er noch immer als Sachwalter des Krieges gilt. In der Konzeption der Inneren Führung finden sich - zumindest im Ansatz - viele der nachfolgenden Gedanken. Das ist kein Zufall, war diese doch von Anbeginn auf Friedenserhaltung angelegt. Als Grundvoraussetzungen für einen nicht-gewalttätigen Austrag zwischenmenschlicher Spannungen wurden Freiheit und Recht zu Maßstäben der Menschenführung gesetzt. ${ }^{49}$

Die Gedanken zu Frieden auf der anderen Seite aus politischer Sicht entwickelte Baudissin in der Folgezeit politisch- und militär-strategisch fort. Der eine Ausgangspunkt dazu ist sein Verständnis vom künftigen Krieg, den er als »permanenten Bürgerkrieg « bezeichnete und der - wie gesagt - keine räumlichen, zeitlichen oder sachlichen Abgrenzungen mehr ken-

\footnotetext{
45 In dieser Quelle Baudissin 52,5; ders. 1969, S. 140 heißt es »autonom«, an anderen Stellen spricht Baudissin in diesen Jahren vom »absoluten Krieg «. 46 Baudissin 52,5; ders. 1969, S. 140.

47 Baudissin 53,8; ders. 1969, S. 143.

48 Baudissin 58,6.

49 Baudissin 68,8; ders. 1969, S. 28.
}

ne. ${ }^{50}$ Wesentlich bestimmt sei dieser davon, »dass hier der andere gezwungen wird oder meint, zu einer Kampfweise gezwungen zu sein, die im Grunde genommen nicht die Seine ist $\varkappa^{51}$. sowie: $»$ Allgemeine Friedlosigkeit ist das Kennzeichen dieser Auseinandersetzung, die auf allen Lebensgebieten ausgetragen wird.« und weiter: der Soldat sei »in ganz besonderem Maße Ziel, Mittel und Träger dieser Auseinandersetzung, obwohl die militärische Verteidigung nur noch eine Form des Schutzes nach außen und nicht einmal die aussichtsreichste darstellt $\ll^{52}$. Mehr fragend und dennoch bestimmt, stellt er resümierend fest: »Es ist auch in keiner Weise für jeden mehr sicher, dass der Krieg ein vernünftiges Mittel der Politik ist, für eine Anzahl Menschen nicht einmal mehr dann, wenn es um die Verteidigung der durch einen Angriff bedrohten gesamten Existenz geht. ${ }^{53}$

Der andere Ausgangspunkt für Baudissins strategie-bestimmte Überlegungen zur Inneren Führung war das Szenario des Atomkrieges: »Wie weit es im Zeitalter der Atombombe noch Sieger und Besiegte gibt, ist fraglich; und die Aufgabe des Friedens ist mit militärischem Sieg noch nicht gelöst. Ich halte es auch für gefährlich, (einfach so) den militärischen Sieg als letztes Ziel des Krieges hinzustellen, als etwas auch, was ohne weiteres und ohne Folgen zu erwarten ist. ... Er kann nur dann als letzte Möglichkeit gerechtfertigt sein, wenn wir uns gegen einen Gegner verteidigen müssen, der die Existenz der Gemeinschaft und damit zugleich die Existenz jedes Einzelnen zu vernichten droht. Die Streitkräfte sind nach meiner Auffassung kein Instrument mehr dafür, einen aggressiven Willen durchzusetzen, sondern sie sind allein dazu da, dem Gegner durch ein Höchstmaß an Kampftüchtigkeit die Verlagerung der geistigen Auseinandersetzung in die Sphäre des heißen Krieges unratsam erscheinen zu lassen. Dieses Höchstmaß an Kriegstüchtigkeit geht weit über das Technisch-Taktische hinaus. Sie macht es der Politik erst möglich, den Frieden zu bewahren. ${ }^{54}$ In seinem vielbeachteten Aufsatz über das Kriegsbild von 1962 schrieb er: »Soviel dürfte freilich feststehen: hat die Abschreckung erst einmal versagt, so ist die Entwicklung zum Äußersten mehr als wahrscheinlich. Dies liegt im Wesen des Krieges überhaupt, wird aber durch das Risiko eines heutigen und durch das tiefe gegenseitige Misstrauen der Kriegführenden noch erheblich gesteigert. «55 Und zusammenfassend heißt es dort: »Zweck des Krieges ist heute wie ehedem: den

50 Baudissin 51,5; ders. 1969, S. 23.

51 Baudissin 53,18.

52 BMVg 1957a, S. 34ff, hier: S. 35. Bezüglich dieser Funktion des Soldaten sagt er weiter vor dem Sonthofener Lehrgang 1956: »So steht zunächst als soldatische Aufgabe im Vordergrund: Durch ein Höchstmaß an abwehrbereiter Kriegstüchtigkeit Schutz in der latenten Bedrohung zu geben. ... Daß auch dieser Teil der militärischen Aufgabe nur in der Bereitschaft zu erfüllen ist, sich der akuten Drohung mit ganzer Entschlossenheit entgegenzuwerfen, bedarf keiner weiteren Begründung.«Dass, S. 36.

53 Baudissin 53,18.

54 Baudissin 53,18. Vergl. auch derselbe 66,2 in seinem Vorwort zu André Beaufre: Totale Kriegskunst im Frieden, Berlin 1966, ders. 1969, S. 263 ff, in dem er besonders auch Beaufres Vorstellungen zu Frieden und Vorschläge zur Lösung des politisch-strategischen Dilemmas zustimmend aufnimmt.

55 Baudissin 62,2; ders. 1969, S. 69 
Gegner zur Erfüllung des eigenen Willens zu zwingen ${ }^{56}$. Daran ändert auch die Verlagerung der Auseinandersetzung auf die geistig-politische Ebene nichts. `Kriegsziel` der freien Welt ist es, zumindest militärisch, den Frieden zu erhalten und jedes Antasten der freiheitlichen Ordnung zu verhindern. So bleiben auch die Streitkräfte ein Mittel der Politik, aber eben nur noch eines im politischen Krieg neben Wirtschaft, Recht, Gesellschaft, Technik und Wissenschaft; sie entwickeln ihren höchsten politischen Wert, wenn sie abschrecken, ohne eingesetzt zu werden. $\ll^{57}$

Die starke Bedeutung von Frieden für Baudissins Arbeiten dieser Jahre steht außer Zweifel. Er spitzt seine Gedanken für die Führungspraxis in den Streitkräften sowie für deren politischmilitärische Strategie sogar in einer Weise zu, dass manche Mitstreiter ihm dabei nicht folgen können. Das gilt sowohl bei der Formulierung des Bildes vom »Soldat für den Frieden « als auch für die Feststellung, dass Krieg kein vernünftiges Mittel der Politik sei, sondern Streitkräfte die Aufgabe haben, den Frieden zu bewahren. Dieser apodiktische Ausschluss jeglichen politischen Grundes für Krieg - ausgenommen der Abwehr von Aggressionen - beinhaltet das Paradoxon, dass Friede nur durch höchste Kriegstüchtigkeit, militärische Abwehrstärke oder strategisch durch Abschreckung zu erhalten sei. Dieser zweite Gedanke scheint wieder mit Brunners Überlegungen zur Friedensordnung in Verbindung zu stehen. Da Baudissin aber den von Brunner betonten Friedenswillen nicht mit anspricht, müssen wir hier eine weitere Konkretisierung des praxisorientierten Ansatzes zum Umgang mit - auch von Brunner so gesehenen - »Friedensstörern « bei Baudissin feststellen.

Baudissins Bild von der friedenspolitischen Großwetterlage scheint sich in dieser Zeit jedoch im Sinne des berufsbezogenen Verwertungsinteresses deutlich verschoben zu haben. 1947, bei der Lektüre von Brunner, konnte er mit diesem allgemein von einer Sehnsucht nach Frieden unter den Völkern ausgehen. Jetzt scheint für ihn Frieden alles andere als friedlich zu sein; denn er spricht von allgemeiner Friedlosigkeit sowie von einem permanenten Bürgerkrieg ohne räumliche und zeitliche Grenzen und ohne Beschränkung der Kampfmittel.

Der bereits in der Zeit vorher von Baudissin entwickelte doppelte Zugang zu Frieden aus individueller und aus politischer Sicht wird von ihm im Hinblick auf die praktischen Anforderungen seiner militärischen Aufgaben noch stärker herausgearbeitet. Dadurch entsteht für ihn deutlicher als vorher die Frage der Vermittlung beider Bereiche. Die Antwort darauf beinhaltet sowohl individuelle als auch politische Lösungsmöglichkeiten: Zum einen das mitmenschliche friedliche, angstfreie Verhältnis und einen nicht-gewalttätigen Austrag zwischenmenschlicher Spannung, was gegebenenfalls durch

56 Diese Maxime entwickelte Baudissin sieben Jahre später weiter: »Nach wie vor gilt das Wort von Clausewitz, dass es Ziel des Krieges ist, dem Gegner den eigenen politischen Willen aufzuzwingen. Nur, dass der politische Wille nicht mehr darauf abzielt, dem anderen etwas aufzuzwingen, was dessen politische Existenz bedroht. Es geht heute darum, sich dem Willen des Angreifers nicht selbst zu unterwerfen; nicht mehr um `Siegen' geht es, sondern um >Nicht-besiegt-Werden'. "Baudissin 68,8; ders. 1969, S. 39. Vrgl. ders 68,3 ders. 1969, S. 293f: Es gehe nicht um das »militärische Siegen - dies diene keinem politischen Zweck -. »Das militärische Nicht-Besiegt-Werden wird häufig genügen, um den Angreifer zu hindern, seinen politischen Willen dem Angegriffenen aufzuzwingen. Ein solcher 'Sieg، genügt.«

57 Ebd., S. 75.
Erziehung oder anders: durch politische Bildung zu befördern ist. Und zum anderen eine neue politisch-geistige und gesellschaftliche Ordnung. In diesem Zusammenhang entwickelt er auch sein Verständnis von Frieden fort hin zu einem positiven Friedensbegriff, wenn er als politisches Ziel von »Stärkung und Wahrung des Friedens « spricht. Hier klingen wieder Gedanken von Brunner an.

\section{Frieden in Baudissins friedensrelevanten Vor- stellungen als Friedensforscher 1968-1984}

Baudissin begann im Frühjahr 1968 - bereits kurz nach seinem Ausscheiden aus dem militärischen Dienst - als Lehrbeauftragter an der Hamburger Universität Vorlesungen zu halten. Sein erstes Thema war »NATO-Strategie im Wandel «. ${ }^{58}$ Die Frage nach dem Kriegsbild ist gleichermaßen Ausgangspunkt wie Ziel seiner Überlegungen, an dem »Friedensstreitkräfte « mit deren Strategie und Taktik zu orientieren seien. Mit Aron spricht er vom »Ohnmachtsfriede ${ }^{59}$, »in dem gegenseitige Abschreckung die dialektische Situation schafft, dass eine nie erlebte Machtfülle den Weltmächten gleichzeitig Beschränkungen in der Machtanwendung auferlegt«. Seine weiteren Ausführungen markieren einen Übergang von Vorstellungen der Kriegsverhinderung mit entsprechender politisch-militärischer Strategie der Abschreckung hin zu friedenspolitischen Fragen nach einer politischen Strategie der Friedenserhaltung und einer dementsprechenden beständigen Friedensordnung.

Damit betrat er den damals in der deutschen Gesellschaft neuen Weg der Friedensforschung. Sein Ansatz für Friedensforschung ist von Anfang an auf Politik-Beratung ausgerichtet. Er beschreibt ihn als »pragmatische Schule «:

»Die Friedens- und Konfliktforschung aller Richtungen geht davon aus, dass der Krieg weder ein Naturgesetz noch ein notwendiger Bestandteil menschlichen Lebens ist, sondern das Ergebnis ganz bestimmter Haltungen, Erwartungen und Handlungen. Damit muss es möglich sein, den Krieg zu überwinden wie weiland Kannibalismus und Sklaverei,

- die pragmatische Schule meint, dass harte Interessengegensätze zum menschlichen Leben gehören, dass es daher nicht um Abschaffung, Ächtung oder Verdammung von Konflikten gehen kann, sondern um ihre Rationalisierung, wenn Sie so wollen, um ihre Humanisierung, also um ihren gewaltfreien Austrag nach gegebenen Normen und Regeln. ${ }^{60}$

58 Ders. 68,3. In überarbeiteter Form ist dies als »NATO-Strategie im Zeichen der Friedenserhaltung « in ders. 1969, S. 267-302ff abgedruckt.

59 In ders. 72,9 heißt es dazu: »Die militärische Abschreckung ist zwar imstande, mit einiger Wahrscheinlichkeit Nichtkrieg zu halten, nicht aber, den Frieden zu gestalten. Das ist Aufgabe der Politik, die den strategisch stabilen Zustand zu Entspannungspolitik und Friedensgestaltung nutzen muss.

60 Diesen Gedanken hatte er bereits 1972 anlässlich der Rede zum Volkstrauertag in Flensburg formuliert, s. ders. 72,27:» Wer aber Gewaltanwendung - und damit auch Krieg - in dieser spannungsgeladenen Welt ablehnt, muss auf andere Mittel zur lebensnotwendigen Konfliktregelung sinnen, muss Strukturen und Mechanismen gewaltfreien Konfliktaustrags anbieten. Die entscheidenden Voraussetzungen für das Funktionieren solcher Regelungen liegen freilich im Menschen selbst. Nur wenn es den Menschen gelingt, in sich selber friedlichere Haltungen und Verhaltensweisen zu entwickeln, nur dann können auch Krieg und Gewalt überwunden werden.« 
- Erziehung zum Frieden ist also nichts anderes als Erziehung zur Konfliktfähigkeit. Diese Erreichen wir:

- wenn wir anerkennen, dass zu unserem Leben Konflikte aller Art in Familie, in kleineren wie größeren Gruppen, in der Gesellschaft und zwischen Staaten gehören;

- wenn wir in demjenigen, mit dem wir Konflikte haben, nicht gleich den Bösewicht sehen, der künstlich Gegensätze aufbaut, sondern jemanden, der genauso subjektiv wie wir selbst bestimmte Interessen und bestimmte Positionen vertritt;

- wenn wir zugeben, dass auch die eigene Position weder absolut, noch subjektiv ist, sondern ebenfalls als relativ zu bewerten ist;

- wenn wir uns darauf einlassen, die Konflikte nicht mehr mit Gewalt zu >lösen', sondern in einem oft frustrierenden Schritt-für-Schritt-Prozess mühsam zu regeln, und begreifen, dass die heute brennenden Spannungen häufig nur dadurch erträglich werden, dass noch brennendere aufflammen.«61

Frieden ist für Baudissin zum einen - noch immer - »bewaffneter Frieden « und »nur relativ und alles andere als konfliktfreies Leben $\aleph^{62}$. Frieden stellt für ihn zum anderen aber die Herausforderung schlechthin dar: »Die Vorstellungen von der Unvermeidbarkeit großer Kriege sind, nach wie vor, virulent - Frieden dagegen erscheint als Utopie. « \$So ist es keine grobe Vereinfachung zu behaupten, dass Frieden heute zur Notwendigkeit geworden ist, sofern die Menschheit sich nicht selbst zerstören, zumindest falls sie unter einigermaßen menschenwürdigen Bedingungen weiter existieren will. «63 Dabei erhalten seine Gedanken eine deutliche Zuspitzung: »In Konsequenz dieser Strategie ist der Soldat auf den Frieden bezogen. Der eigentliche >Feind' ist nicht mehr der Soldat des anderen Bündnisses, sondern der alle Existenz bedrohende Krieg. Den Ernstfall können also nicht mehr Krieg und Kampf bedeuten, die zu verhindern gerade die Aufgabe der Streitkräfte auf beiden Seiten geworden ist. Ernstfall ist hier und jetzt, wenn der Soldat seinen Beitrag zur strategischen Stabilität, d.h. zur Glaubwürdigkeit der Abschreckung durch gewissenhafte Funktionserfüllung im alltäglichen Dienst leistet. « ${ }^{64}$ Damit betonte er das Paradoxon des »Soldat für den Frieden «, das er bereits 1956 vor dem Sonthofener Lehrgang formuliert hatte, dass die Erhaltung des Friedens von der Bereitschaft zum Krieg abhängt. Als eine Konsequenz daraus stellt er den Soldaten entsprechend vor die herausfordernde Frage: »Sollten Soldaten nicht auch den Mut haben, sich heute für die Sache des Friedens zu engagieren, und sollten sie nicht eine lohnende Aufgabe darin finden, im Frieden den Frieden zu bewahren und im Krieg den Rückweg in den Frieden offenzuhalten ...? «65

61 Wolf Graf von Baudissin: Vortrag im Rahmen der Hamburger Universitätstage, 16. - 17. November 1981; MS vom 14.9.1981, S. 3f.

62 Baudissin 76,27. Vergl. auch ders.79,5: »Frieden ist kein konfliktfreier $>\mathrm{Zu}-$ stand', der sich durch verbale Ächtung von Krieg und Gewalt ein für allemal herbeizaubern lässt. Frieden ist ein ungewöhnlich dynamisches Geschehen «.

63 Baudissin 1969, S. 32 und 35

64 Baudissin 71,18; s.a. ders. 1982, S. 148. - Das Wort »Frieden ist der Ernstfall geht auf den dialektischen Theologen Karl Barth zurück, s. ders.: Kirchliche Dogmatik Band III/4: Ethik der Schöpfungslehre, Es hat durch den späteren Bundespräsidenten Gustav Heinemann allgemein-gesellschaftliche Bedeutung erlangt.

65 Baudissin 69,9.
Sicherheitspolitik hat für Baudissin ausschließlich eine »friedensbewahrende Zielsetzung «. »Ihr Mittel ist strategische Stabilität; sie wird durch die Militärstrategien gegenseitiger Abschreckung und kooperativer Rüstungssteuerung gewährleistet. Streitkräfte üben unter diesen Bedingungen eine lediglich prohibitive Funktion aus. $« 66$

Die Aufgabe der Sicherheitspolitik bestehe darin, Symmetrie des militärischen Kräfteverhältnisses herzustellen und zu erhalten. Dabei gehe es nicht um »klare Symmetrien in allen Ebenen, Sektoren oder Systemkategorien «, sondern um eine »belastbare Gesamtstabilität«, die für jeden Beteiligten aus einem »kalkuliert untragbares Risiko « entstehe. ${ }^{67}$ Der Entspannungsprozess verlange darüber hinaus, Instabilitäten der anderen Seite ernst zu nehmen und bei ihrem Abbau zu helfen. Das fordere auch »die Bereitschaft, Interdependenzen einzugehen, also Einbußen an klassischer Souveränität hinzunehmen « und mache Vertrauensbildende Maßnahmen bei Kooperativer Rüstungssteuerung notwendig. ${ }^{68}$

Entscheidend sei dafür die Erkenntnis, dass beide KonfliktSeiten aufeinander angewiesen sind: »Beide Seiten sind dazu verurteilt, sich mit der Dynamik und der Belastung des dialektischen Prozesses abzufinden, der zwischen den Leitplanken Annäherung und Abgrenzung verläuft. Die Annäherung dient der Stabilisierung der Systeme durch erhöhte Kooperation, die Abgrenzung durch verschärfte Kontrolle der Gesellschaft und Wiederbelebung des Feindbildes. « Dies verlange einen beiderseitigen Lernprozess: »Es geht darum, in dem Gegenüber nicht allein den Antagonisten, sondern zugleich den Partner zu erkennen, ohne dessen Kooperation immer weniger Probleme geregelt werden können. « ${ }^{69}$ Im Interview mit Cornelia Bührle fasst Baudissin entsprechend zusammen: »Die Schaffung friedlicherer Beziehungen setzt freilich vor allem ein neues Verhältnis zu Konflikten im allgemeinen, zu Opponenten und zur eigenen Position im besonderen voraus. Eine Rationalisierung menschlicher Beziehungen ermöglicht es, die vielerlei Spannungen als unabänderliche Fakten zu akzeptieren. Sie erlaubt es, sich geduldig auf Regelungsprozesse einzulassen, anstatt schnelle und definitive Lösungen zu erwarten; sie befähigt uns schließlich dazu, den jeweiligen Kontrahenten nicht mehr als den Störenfried oder gar Bösewicht zu betrachten, sondern als jemanden, der seine andersgearteten Interessen mit gleicher Berechtigung vertritt wie wir die unseren. Eine solche Relativierung der Positionen entschärft und humanisiert die Beziehungen; sie gibt den Weg frei zu schrittweise Regelungen von Differenzen. ${ }^{70}$

Baudissin verknüpft diese politischen Aspekte zu Frieden wieder mit individuellen, wenn er sagt: »Die Gestaltung einer friedlicheren Zukunft ist eine Aufgabe, die wir nicht den anderen, auch nicht den politischen Machtträgern überlassen dürfen. Der Krieg und damit auch der Frieden beginnt im Her-

66 Baudissin 76,27, S. 2.

67 Baudissin 76,1; ders. 76,28. In der Denkschrift von 1947 spricht Baudissin vom »unverantwortbaren Risiko«.

68 Wolf Graf von Baudissin: Bemerkungen zu den Heidelberger Thesen. In Detlef Bald (Hrsg.): Europäische Friedenspolitik - Ethische Aufgaben. BadenBaden 1990, S. 31-34, hier: S. 32.

69 Baudissin 76,28

70 Baudissin 81,43 
zen des einzelnen. «71 Dieser müsse deswegen konfliktfähig, d.h. sich der Subjektivität der eigenen Vorstellung bewusst und daher tolerant und zu Kompromissen bereit sein. Anders ausgedrückt: »All das fordert Selbstüberwindung, Engagement, Zivilcourage und einen erheblichen Schuss Optimismus - um nicht zu sagen: Liebe. $\ll^{72}$

An seinem Lebensabend - bei der Auseinandersetzung mit den Heidelberger Thesen von 1959 - fasste Baudissin diese Gedanken in drei Möglichkeiten zur Überwindung von Konflikt zusammen $^{73}$ :

Als erstes eine Geisteshaltung, die mit sachlicher Offenheit für das Gegenüber zu beschreiben wäre. Sie begründet sich für Baudissin darin, dass es nur eine "gemeinsame Sicherheit « gebe: »Das Sicherheitsbedürfnis des Gegenüber ist zu einem wesentlichen Element der eigenen Sicherheit geworden. ${ }^{74}$ Dies schließt an die Forderung nach moralisch-praktischer Souveränitätsbegrenzung an: »Der Entspannungsprozess verlangt darüber hinaus, Instabilitäten der anderen Seite ernstzunehmen und bei ihrem Abbau zu helfen. ... Wichtig wird die Bereitschaft, Interdependenzen einzugehen, also Einbußen an klassischer Souveränität hinzunehmen.«

Als zweites eine menschliche Qualität der Handelnden, die sich für Baudissin aus dem Verständnis von Konflikt - generell wie auch politisch - ergibt: »Frieden scheint mir primär Konfliktfähigkeit zu fordern. Diese verlangt zunächst einmal die Anerkennung der bitteren Tatsache, dass Konflikte Phänomene menschlicher Existenz sind und auf allen Ebenen unseres Daseins auftreten. ... Wer eine friedliche Regelung der Konflikte anstrebt, sollte zumindest vor sich selbst die Bedingtheit auch der eigenen Position zugeben und der Gegenseite mit Empathie und Verständnis begegnen, ihr also mit christlicher Nächstenliebe und Verständnis gegenüberstehen.«

Und schließlich - unter Hinweis auf einen transzendentalen Bezug - die zusätzliche Forderung an den Handelnden nach Realitätssinn wie Selbstbeschränkung: »Gottvertrauen sollte optimistisch stimmen, die Nächstenliebe Konfliktfähigkeit entwickeln helfen und das Wissen von der Bedingtheit allen menschlichen Tuns sie vor idealistischen, sprich ideologischen Wunschträumen bewahren. $\ll^{75}$

Der Forschungsschwerpunkt Frieden führt Baudissin augenscheinlich wieder näher an Gedankengänge, die er bei Brunner kennen gelernt hatte. Dies wird bereits deutlich an der Betonung einer freiwilligen Beschränkung der klassischen Staaten-Souveränität sowie des individuellen Vertrauens z. B. als Toleranz, Offenheit, Verständnis oder Empathie, als auch des politisch-gesellschaftlichen Vertrauens z. B. als »Vertrauenbildende Maßnahmen « oder dem Bewusstsein, aufeinander angewiesen zu sein. Auffällig ist auch die Benutzung der Begriffe Störenfried und Entspannungswille. Besonders springt die Nähe zu Brunner ins Auge bei der Betonung von Liebe und Nächstenliebe; diese hat zwar bei Brunner keinen direkten Bezug zu seinen Ausführungen über Frieden; das von ihm in

71 Baudissin 72,27

72 Dass.; vrgl. a. Baudissin 79,5.

73 Baudissin 1990, S. 32ff.

74 Baudissin 72,9.

75 S.a. Baudissin $68,8$.
»Gerechtigkeit « entwickelte Verständnis von Liebe aus christlicher Sicht wird jedoch von Baudissin vollkommen geteilt und hier in seine Überlegungen zu Frieden als Letzt-Forderung an das Individuum eingeführt.

Auch die Herangehensweise an die Frage nach Möglichkeiten zur Bewahrung, ggf. Wiederherstellung sowie zur Gestaltung des Friedens, ausgehend von einer Extrem- oder Grenzbetrachtung her, weist auf Brunner, der diesbezüglich den fiktiven Urzustand der Anarchie sowie das seiner Zeit aktuelle Bedürfnis nach Frieden betont hatte. Baudissins Grenz-Gedanken sind jedoch eher sach- und auch stärker zukunftsbezogen, wenn er von Krieg als dem beiderseitigen Feind und von »kalkuliert untragbarem Risiko« spricht.

Die bei Baudissin immer schon beobachtete eher auf politische Praxis ausgerichtete Sichtweise wird auch hier wieder deutlich. Das schon 1950 formulierte »Schwert als Damm« nimmt er als politische Strategie der Abschreckung im Rahmen von Sicherheitspolitik auf. Zum anderen ist er davon überzeugt, dass Frieden auf Erden machbar ist, wenn auch nur in kleinen Schritten, sprich: durch gewaltfreie Zwischenregelungen. Daraus entwickelt er seine Überlegungen zur "präventiven Kooperativen Rüstungssteuerung « - so der von ihm anstelle von arms control eingeführte Begriff - als die ergänzende Strategie der Sicherheitspolitik. Deren Einzelaspekte wie einseitige Schritte, relative Stabilität oder belastbare Gesamtstabilität erinnern ebenfalls wieder an Brunners Forderung nach »moralisch-praktischer Souveränitätsbegrenzung«.

\section{Schluss}

Die Antwort auf die generelle Ausgangsfrage nach der Bedeutung von Baudissins gedanklichen Auseinandersetzungen mit der »Schicksalsfrage « aus seiner Zeit in der Gefangenschaft für seine späteren Arbeiten zur Inneren Führung und Friedensforschung und speziell, wie das Thema Frieden für Baudissins weiteres Denken und Handeln die hervorragende und bestimmende Kategorie geworden ist, lautet zum einen allgemein: Die Arbeit an seiner Denkschrift »Ost oder West « ist der geistige Wendepunkt zur Neuorientierung in seinem Lebenswerk in Gesellschaft und Politik, für Strategie sowie für die Streitkräfte und Soldaten der Bundeswehr. Und zum anderen konkret zum Thema Frieden: Baudissin hat in Brunners »Gerechtigkeit « den entscheidenden Anstoß erfahren, das Thema Frieden insgesamt für sich neu und als revolutionär zu entdecken. Brunners Gedanken über Frieden sind der generelle Denkanstoß zu Baudissins Friedensorientierung, die Initialzündung zu seiner Beschäftigung mit dem Thema Frieden; denn bis zur Lektüre von »Gerechtigkeit« war Baudissins Suche nach Neuorientierung für die Zeit nach dem geistig-sittlichen Zusammenbruch inhaltlich ohne die Frage nach Frieden ausgekommen. Von nun an bis an sein Lebensende ist Frieden für Baudissin die bestimmende normative und sachliche Denkkategorie, sie ist für ihn gesellschaftlich-politisch ohne Alternative und eine individuelle Herausforderung an jedermann. Frieden ist der Ernstfall des Lebens. 
Dabei ist zu sagen: Baudissin hat Brunner nach 1947 nie wieder in seinen Reden und Schriften zitiert oder namentlich erwähnt, so dass Verbindungen zwischen Baudissins Aussagen über Frieden und denen von Brunner nicht eindeutig zu belegen sind. Das Buch »Gerechtigkeit« hat für Baudissins weiteres Wirken und seine konkreten Arbeiten auch im Detail aber grundlegende Bedeutung erlangt. Es hat ihm - je nach Verwertungsinteresse fortentwickelt und ausgearbeitet - ein umfangreiches Angebot von neuen Gedanken für viele seiner späteren detaillierten Überlegungen zu einem friedlichen Umgang der Völker miteinander abgegeben. Bereits 1947 finden wir in Baudissins mehr praktisch orientierten Gedanken zu Frieden viele Einzelüberlegungen von Brunner. War Frieden für ihn dann im Rahmen seiner Arbeiten in Bundeswehr und NATO jedoch mehr ein letzter normativer und ethischer Bezugspunkt für Handeln in Politik sowie mit und von Streitkräften, so kennzeichnen ein sachlich-rationales Herangehen an den Gegenstand Frieden und dessen differenzierte Behandlung - soweit Brunner ähnlich - die Ausführungen des Friedensforschers Baudissin. Dabei führt ihn das Grundverständnis von Paradoxa, Gegensätzen und Konflikt mit der Suche nach und Betonung von Gemeinsamkeiten »um des Besseren willen « zu einem neuartigen, einem sozialen Verständnis von Frieden und Krieg als höchst dynamisches Verhältnis, wie wir es bei Brunner nicht erkennen können. Ansätze dazu, die in dessen Gedanken über Friedenswille und moralisch-praktische Souveränitätsbegrenzung zu finden sind, mag Baudissin bei seiner eklektischen Aufbereitung von Brunners »Gerechtigkeit « empfunden und dann weiter ausgearbeitet haben. Ebenso wird Baudissin bei der Lektüre von Brunners »Gerechtigkeit « dessen deutlich getrennte Betrachtungen nach allgemeinen politischen sowie nach individuellen Aspekten aufgenommen haben. Er hat diese dann aber bereits in seinen Vorträgen um 1950 - über Brunner hinausgehend - auf die Behandlung von Frieden übertragen und betont miteinander verknüpft. Daraus hat er im weiteren Verlauf seiner Arbeit einerseits sozialpsychologisch praktische politische Maßnahmen wie Vertrauen bei Vertrauensbildenden Maßnahmen oder Kooperieren bei Kooperativer Rüstungssteuerung entwickelt und andererseits individuell Nächstenliebe und Liebe als letzte tiefe Handlungsnormen aus christlich-ethischer Sicht für jedermann - auch in der Politik - aufgestellt. Nicht zuletzt sieht er wie Brunner für politische Bildung eine große Aufgabe zur Entwicklung von Friedensfähigkeit der Akteure.

Was bedeuten diese Ergebnisse nun für uns heute über das rein historische Interesse der Wirkungsmacht von Brunners Gedanken auf Baudissins Werk hinaus? Sie beinhalten eine Botschaft, die heute wieder wie damals 1943-1947 aktuell - vielleicht sogar noch aktueller - ist und von daher bei der derzeitigen Neuorientierung von Politik und Reform der Streitkräfte aufzunehmen ist:

- Frieden aus christlich-ethischer Sicht ist der normative Orientierungspunkt für Handeln und Erwarten in Gesellschaft und Politik, für Strategie sowie für die Streitkräfte und Soldaten der Bundeswehr.

- Frieden ist nicht nur eine reale Utopie, sondern der Ernstfall und machbar.

- Die Verknüpfung von individuellen und politisch-sozialpsychologischen Aspekten bietet praktische Handlungsmöglichkeiten im Rahmen einer friedensorientierten Sicherheitspolitik mit entsprechender politisch-militärischer Strategie sowie in Führungsstrukturen und -prozessen der Streitkräfte.

- Das christlich-soziale Verständnis von Konflikt bietet praktische Möglichkeiten zur Behandlung nicht nur von individuell-zwischenmenschlichen Konflikten in überschaubaren Gruppen und Organisationen, sondern auch von gesellschaftlich-politischen im zwischengesellschaftlichen bzw. zwischenstaatlichen Rahmen.

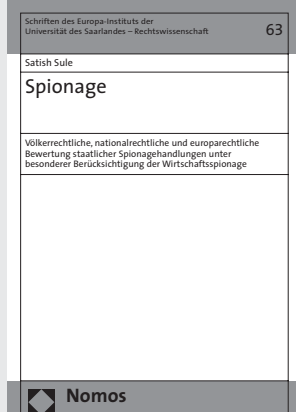

\section{Spionage}

Völkerrechtliche, nationalrechtliche und europarechtliche Bewertung staatlicher Spionagehandlungen unter besonderer Berücksichtigung der Wirtschaftsspionage

Von Dr. Satish Sule, LL.M. Eur.

2006, 430 S., brosch., 78,- $€$, ISBN 3-8329-1756-X

(Schriften des Europa-Instituts der Universität des Saarlandes - Rechtswissenschaft, Bd. 63)

Die Untersuchung nimmt eine umfassende rechtliche Bewertung staatlicher Spionagehandlungen auf völkerrechtlicher und nationalrechtlicher Ebene - aus deutscher, französischer und britischer Sicht - vor. Das Kernstück der Arbeit bildet die erstmalige europarechtliche Untersuchung mitgliedstaatlicher Spionage unter besonderer Berücksichtigung der Wirtschaftsspionage. 